\title{
Germanica
}

\section{Bühler et la subjectivité}

Bühler und die Subjektivität

\section{André Rousseau}

\section{CpenEdition}

Journals

Édition électronique

URL : http://journals.openedition.org/germanica/2419

DOI : 10.4000/germanica.2419

ISSN : 2107-0784

Éditeur

Université de Lille

\section{Édition imprimée}

Date de publication : 30 juin 2000

Pagination : 163-184

ISBN : 9782913857032

ISSN : 0984-2632

Référence électronique

André Rousseau, "Bühler et la subjectivité », Germanica [En ligne], 26 | 2000, mis en ligne le 11 mars

2014, consulté le 06 octobre 2020. URL : http://journals.openedition.org/germanica/2419; DOI :

https://doi.org/10.4000/germanica.2419

Ce document a été généré automatiquement le 6 octobre 2020.

(c) Tous droits réservés 


\title{
Bühler et la subjectivité
}

\author{
Bühler und die Subjektivität
}

André Rousseau

1 Karl Bühler (1879-1963) occupe une place tout à fait à part sur l'échiquier de ce que l'on n'appelait pas encore les « sciences humaines » ou « sciences de l'homme ». Après avoir fait des études de médecine, ponctuées par un doctorat en 1903 à Fribourg et ensuite de psychologie, avec un nouveau doctorat en 1904 à Strasbourg, il entra dès 1906 dans le cercle d'O. Külpe, dit " école de Würzburg ", où il soutint son habilitation en 1907 sur un ouvrage intitulé $\mathrm{Zu}$ einer Psychologie der Denkvorgänge. Il a donc été le disciple du fondateur de la " psychologie de la pensée » en Allemagne, Oswald Külpe (1862-1915), avant de devenir lui-même le maître incontesté de cette spécialité.

2 Il suivit son maître et bientôt son ami Oswald Külpe à Bonn, puis à Munich ; il eut dans les années 1907-1908 de vives discussions avec W. Wundt, dont il combattait le psychologisme et l'associationnisme. À la mort de Külpe en 1915, il reprit, en pleine guerre, la direction de l'Institut de Psychologie. Après un assez bref passage à Dresde, il obtint en 1922 une chaire à Vienne, la ville de S. Freud, où il séjournera seize ans. C'est évidemment la période la plus pleine et la plus créatrice de la vie professionnelle du couple Bühler, car il avait épousé Charlotte en 1916, psychologue elle aussi et ancienne élève de Husserl. Mais en 1938, à la suite de l'arrivée des nazis à Vienne, il doit fuir l'Autriche et se réfugie avec Charlotte en Norvège, avant de gagner définitivement les États-Unis où il restera jusqu'à sa mort en 1963.

Bühler possédait une immense culture aussi bien en philosophie (à commencer par Platon et Aristote) qu'en psychologie, sa spécialité majeure, qu'il a constamment enseignée, et en linguistique, discipline qu'il pratiquait intensément et qui l'avait amené à accueillir par ex. J. Kurylowicz dans son laboratoire.

4 Il y a certainement deux manières de lire et d'interpréter la Sprachtheorie (1934), son ouvrage sur le langage, qui contient ses thèses majeures : soit en la replaçant dans le continuum de ses travaux, notamment à la suite de sa Krise der Psychologie (1927); soit en la situant dans le contexte linguistique des années 30. L'une ou l'autre lecture mettra l'accent sur des points différents de l'œuvre ou peut-être sur les mêmes points, mais 
avec un éclairage différent. Le présent article essaiera avant tout de présenter une synthèse de sa doctrine de la subjectivité, sans s'interdire les comparaisons avec d'autres auteurs et d'autres ouvrages de Bühler.

5 Cependant, malgré des publications récentes sur son œuvre (Graumann/Herrmann, 1984 ; von Eschbach, 1988 ; Bühler-Studien, 1984), la pensée de Bühler est encore loin de connaître le retentissement qui aurait dû être le sien. Certes, il est présent en France dès 1933 dans un ouvrage collectif sur la Psychologie du Langage, il est mentionné en 1947 dans un ouvrage de Piaget et son nom est évoqué ici ou là (M. Rossi, 1977). On peut aussi estimer qu'il a nourri plusieurs articles de Benveniste sur la sémiologie et la subjectivité dans le langage. Mais C. Kerbrat-Orecchioni ne mentionne même pas son nom dans un ouvrage ayant pourtant pour titre L'Énonciation. De la subjectivité dans le langage (1980).

6 Ces quelques remarques prouvent qu'au fond la situation n'a guère changé depuis 1934 : même si le modèle de l'Organon est assez souvent cité dans divers ouvrages, c'est en fait une reprise quasi mécanique d'auteur à auteur et plus de soixante ans après leur parution, les thèses de Bühler, qui font l'originalité de sa Sprachtheorie, ne sont toujours pas vraiment connues, ni exploitées, ni continuées.

7 Le présent article abordera trois questions essentielles : les grandes thèses de Bühler, telles qu'elles figurent dans l'Axiomatique; sa théorie de la deixis, qui est la première en date sur le sujet ; enfin, sa théorie de la subjectivité dans le langage, avec une attention particulière à la littérature.

\section{Les grandes thèses de Bühler}

8 Ses grandes thèses ont fait l'objet d'une Axiomatique, publiée d'abord en 1933. Comment Bühler en est-il venu à cette publication ? Quel est le sens de sa démarche?

9 À côté de sa spécialité en psychologie de la pensée, de la perception et du développement, Bühler a toujours manifesté un intérêt jamais démenti pour les questions touchant la théorie du langage. Commençant comme Jakobson par les problèmes concernant l'aphasie, le langage enfantin et le langage des animaux, il fut attiré ensuite par les problèmes fondamentaux soulevés par ces questions en apparence marginales et auxquels pouvait seule répondre une axiomatique. Son premier article d'importance s'intitule Kritische Musterung der neueren Theorien des Satzes (1920), où à partir de l'Organon il conçoit les trois fonctions du langage, qu'il ne cesse de reprendre et de développer dans divers congrès, articles ou comptes-rendus.

Déçu par la psychologie de son époque, comme il le manifeste dans sa Krise der Psychologie (1927), il cherche à établir un pont entre psychologie et langage, puis à construire un édifice théorique dans lequel il pense trouver les réponses adéquates aux problèmes posés par l'expérience, l'expression, le comportement. C'est exactement l'objectif qu'il se fixe dans son Axiomatik der Sprachwissenschaften (1933), reprises avec quelques modifications mineures l'année suivante dans sa Sprachtheorie.

11 Il faut souligner un aspect très moderne chez Bühler, qui n'allait absolument pas de soi à son époque. Il a mis en lumière deux exigences fondamentales pour le théoricien : rendre compte des faits observés dans les langues (c'est ici le psychologue qui parle) et proposer un ensemble cohérent de thèses générales sur le fonctionnement du langage humain (c'est la voix de l'épistémologue) avec un continuel va-et-vient entre les deux 
exigences, l'une soutenant l'autre et réciproquement. En d'autres termes, la description linguistique doit être à la fois empirique et théorique et procéder aussi bien par induction que par déduction.

12 Bien qu'il s'agisse d'axiomatique, la présentation de Bühler n'est jamais dogmatique, mais c'est souvent une discussion, toujours argumentée et riche en exemples, avec des positions différentes ou proches des siennes. Nous examinerons les quatre thèses soutenues dans l'Axiomatique dans l'ordre où elles apparaissent dans la Sprachtheorie de 1934.

\subsection{Le modèle de l'Organon}

Reprenant le terme platonicien du Cratyle, Bühler part d'une idée toute simple: le langage est un outil par lequel une personne donnée transmet à une autre personne quelque chose au sujet de choses ou d'état de choses. Les trois éléments constitutifs de l'Organon ou Sinndimensionen (selon une expression de Frege) ne sont pas nouveaux : la Kundgabe existe chez W. Wundt, la Darstellung est présente chez Bolzano et chez Husserl, la Auslösung n'est en revanche qu'évoquée allusivement chez Anton Marty. Mais Bühler explicite la notion de Darstellung par celle de Zuordnung, empruntée aux mathématiques. Il rapporte ensuite les trois fonctions dégagées, auxquelles il donne définitivement les noms de Ausdruck, Appell et Darstellung, aux trois personnes du discours: $\mathrm{je}, \mathrm{tu}, \mathrm{il} / \mathrm{elll}$. La reconnaissance de ces fonctions soulève une question importante : le signe possède-t-il ces fonctions en quelque sorte intrinsèquement ou bien est-ce l'intention du locuteur et la présence d'un contexte adéquat qui l'orientent vers telle ou telle fonction? Il semble évident que ces fonctions sont chez Bühler des fonctions liées aux types d'énonciation dans une situation concrète donnée.

On se plaît souvent à rappeler que Bühler publie sa Sprachtheorie deux ans seulement après la Theory of Speech and Language (1932) d'Alan H. Gardiner. Mais c'est oublier que dès 1920, dans sa Kritische Musterung der neueren Theorien des Satzes, Bühler avait annoncé: "La fonction du langage humain est triple: Kundgabe, Auslösung und Darstellung », comme il le rappelle lui-même en note en 1933 (1933: 74 note). En tout cas, l'écho est immédiat: W. Troubetzkoy, son collègue slavisant de l'Université de Vienne et du Cercle Linguistique de Prague, en fait le fondement de la division ternaire de sa phonologie, telle qu'elle paraittra plus tard dans les Grundzüge der Phonologie (1938).

15 À bien considérer la position exprimée notamment par Bühler en 1933 dans Ausdruckstheorie, on ne peut s'empêcher de penser à une interprétation plus profonde des trois fonctions identifiées par Bühler. En effet, dans l'interprétation usuelle, ces trois fonctions sont toujours rapportées successivement à l'un ou l'autre des trois pôles du triangle : 


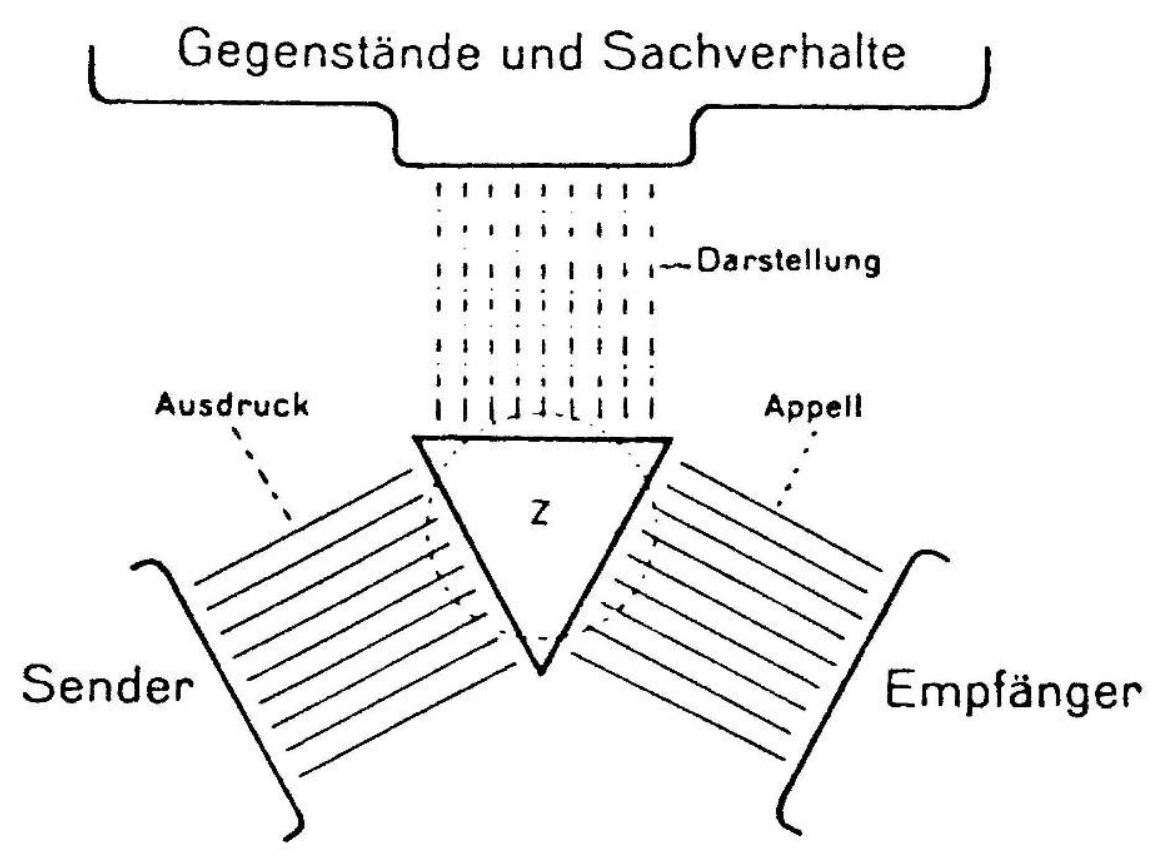

$(1934: 28)$

16 Mais la position de Bühler nous semble plus complexe et plus subtile. Comme le montrent à l'évidence les traits tracés par Bühler, l'essentiel de son schéma concerne les relations entre les angles, que l'on peut hiérarchiser ainsi :

17 1) la relation locuteur-auditeur est une relation d'interlocution, c'est-à-dire d'intersubjectivité, notion qui apparaît plusieurs fois dans l'ouvrage et qui est la garantie essentielle de l'objectivité du contenu linguistique du message ;

2) par l'existence de l'objectivité, issue de l'interlocution au sein d'une communauté linguistique, le langage accède à cette fonction de représentation - qui est bien la fonction fondamentale, comme le souligne le sous-titre de l'ouvrage (Die Darstellungsfunktion).

\subsection{Un " système de signes »}

19 Le second volet de l'Axiomatique de Bühler est constitué par le fait qu'une langue est un « système de signes ». Dans cette expression, l'important pour Bühler - contrairement à Saussure par exemple - n'est pas tant le mot "système ", mais bien davantage le mot "signe» (Zeichen). Il insiste sur le fait qu'un signe représente "quelque chose », au double sens du mot français « représenter », c'est-à-dire à la fois « vertreten » (lat. stare pro) et « darstellen ». La constitution et la reconnaissance d'un tel « système de signes » ressortissent naturellement à l'accord intersubjectif qui crée et valide la convention.

Dans la représentation du «signifiant» (terme saussurien que nous introduisons), Bühler introduit la notion de " pertinence sélective » (abstraktive Relevanz), où seule une partie du complexe de sons exerce effectivement une fonction significative dans le signe. Cette notion de "pertinence sélective» est évidemment issue d'une étroite collaboration intellectuelle avec Troubetzkoy. 
21 Au plan du « signifié » (autre terme saussurien que nous employons), la représentation inclut une notion nouvelle pour l'époque, que Bühler introduit sous le nom d'Umfeld (champ environnnant), ce qui lui permet une prise en compte réaliste de la situation. En effet, Bühler distingue deux types de caractéristiques sémantiques du signe : d'une part, des propriétés inhérentes, appelées feldfremd par Bühler, et d'autre part, des propriétés contextuelles (désignées par feldeigen). Bühler développe ici une analyse extrêmement moderne du signe, qui annonce entre autres les types de sèmes distingués plus tard par B. Pottier.

\subsection{Un modèle organisationnel des champs de l'activité langagière}

Pour Bühler, parler uniquement de " langue » n'a guère de sens, car ce terme est par trop chargé de polysémie. Il est en effet nécessaire de préciser à chaque fois ce que l'on entend par «langue » : soit une force dynamique, soit un état purement statique, soit une ou plusieurs manifestations, soit une faculté inhérente à l'homme. Et d'ailleurs, les grands théoriciens ont bien senti cette nécessité d'obtenir clarté et lisibilité en procédant à un découpage du champ linguistique : ainsi W. von Humboldt a proposé le couple « energeia » - « ergon » et Saussure la dichotomie « parole » - « langue ».

Pour sa part, Bühler propose une sorte de croisement entre ces deux oppositions comme l'a bien remarqué Laziczius (1939) - sous forme d'une table à double entrée :

\begin{tabular}{|l|l|}
\hline $\begin{array}{l}\text { Sprechhandlung } \\
\text { parole }\end{array}$ & $\begin{array}{l}\text { Sprachwerk } \\
\text { ergon }\end{array}$ \\
\hline $\begin{array}{l}\text { Sprechakt } \\
\text { energeia }\end{array}$ & $\begin{array}{l}\text { Sprachgebilde } \\
\text { langue }\end{array}$ \\
\hline
\end{tabular}

24 Même si cette interprétation reste superficielle et peu satisfaisante (cf. la réponse de Lohmann à Laziczius), il n'en reste pas moins que Bühler oppose nettement dans son organisation du champ linguistique la part de subjectivité à la part d'intersubjectivité, ce qui montre bien une nouvelle fois qu'il s'agit là de l'axe central de son œuvre

\begin{tabular}{|l|l|}
\hline La part de subjectivité & La part d'intersubjectivité \\
\hline Sprechhandlung & Sprechakt \\
爁 & 嘼 \\
Sprachwerk & Sprachgebilde \\
\hline
\end{tabular}

Pour faire le lien avec la terminologie actuelle, on peut constater que si l'énonciation appartient à la «Sprechhandlung» individuelle, la phrase relève du domaine du « Sprachgebilde». 


\subsection{Un système à plusieurs étages} pensée. En effet, pour lui, une langue est fondée sur au moins deux classes d'éléments structurels. Elle possède d'abord un stock d'unités lexicales qu'il dénomme "symboles", qui ont une double capacité : soit de signifier par eux-mêmes, soit de constituer, par associations progressives et grâce aux relations, des "parties de phrase » (i.e. des groupes syntaxiques dans une terminologie plus moderne) et ensuite des phrases - ce qui est le propre de la syntaxe. En d'autres termes, les signes linguistiques disposent par eux-mêmes d'une double valeur: valeur symbolique (Symbolwert) et valeurs contextuelles (Feldwerte). Cette analyse est reconnue parfois sous le nom de dogme du lexique et de la syntaxe (cf. Ausdruckstheorie, 1933 : 71).

La productivité est la caractéristique de systèmes comportant au moins deux classes, comme la langue; cette productivité est exclue des codes en général, où toute combinatoire ou syntaxe est absente. Le " au moins ", qui figure entre parenthèses dans la Sprachtheorie (p.73) a toute son importance quand on sait que Bühler reconnaît également un niveau phonématique sans contenu sémantique, mais doté de la possibilité combinatoire.

On peut ainsi constater que Bühler a découvert deux notions essentielles dans le fonctionnement du langage, dont l'histoire de la linguistique ne lui attribue pourtant jamais la paternité - ce qu'il est nécessaire de réviser dans nos manuels :

1. le débat sur la fameuse «double articulation», objet d'une querelle entre A. Martinet et E. Benveniste, est dorénavant définitivement tranché en faveur de K. Bühler, qui a aussi mis en lumière l'existence d'un niveau phonologique ;

2. la découverte de la productivité infinie de la langue a été trop généreusement attribuée à Chomsky. Certes, nous connaissons déjà les propos de W. von Humboldt : «faire un usage infini de moyens finis »; mais le mécanisme défini par Bühler est encore plus précis: «arriver à représenter à l'aide d'un stock limité de conventions et aussi de structures linguistiques la diversité illimitée de manière suffisamment différenciée et exacte » (1934: 76).

La théorie des deux champs, champ monstratif et champ symbolique, aurait pu elle aussi faire partie de cette Axiomatique; mais comme elle est le véritable objet de l'ouvrage, elle ne peut figurer dans les prémisses.

\section{La théorie de la deixis}

L'étude de la deixis à l'époque de Bühler se situe au croisement de deux courants : l'un issu des néo-grammairiens (Brugmann 1904 ; Bahder 1929) et l'autre ancré dans la sémantique logique dès sa naissance (Frege 1918). Il a l'honnêteté de reconnaître que pour ses thèses décisives le terrain avait été préparé par ses devanciers : ainsi le champ monstratif était-il connu des Grecs et familier aux Wegener, Brugmann et Gardiner. L'examen des déictiques représente un chapitre essentiel chez Bühler de l'étude du fonctionnement du langage et de sa subjectivité, comme il le souligne lui-même en les comparant aux panneaux ou poteaux indicateurs sur les routes: «ils [= les mots monstratifs, all. Zeigwörter] sont subjectifs dans le sens même où tout panneau indicateur donne une indication "subjective ", c'est-à-dire valable et réalisable sans erreur seulement à partir de son emplacement » $(1934: 106)$. 
est le premier à établir une véritable théorie de la deixis, fondée sur une analyse précise du fonctionnement des déictiques dans l'exercice du langage. Il propose de donner comme fondement à toute deixis la notion d'origo, qui sera décisive pour la constitution des "systèmes déictiques " - expression employée pour la première fois par Henri Frei en 1944. Mais l'étude de Bühler comporte une faiblesse : elle est limitée aux seuls "mots déictiques " et, malgré une bonne analyse du morphème latin -badans amabat (1934 : 295), il n'a pas songé à englober les morphèmes verbaux parmi les déictiques. À cet égard, il faudra attendre B. Russell (Signification et vérité, 1940) pour voir enfin les temps verbaux intégrés à la deixis - ce qu'exploitera plus tard E. Benveniste.

\subsection{La déicticité est au cœur du langage}

Le point de départ la réflexion de Bühler, même s'il est situé plus loin dans son ouvrage (1934 : 374-5), est une constatation décisive qu'il fait partager à son lecteur. Il s'attache en effet à montrer que l'ancrage déictique est au fond à l'origine de tout énoncé, aussi banal soit-il : «Si j'entends Il pleut, cela signifie "il pleut là où se trouve le locuteur" ». En effet, l'ajout d'une circonstance a pour effet de briser ce lien déictique congénital : Il pleut au bord du lac de Constance. Et dès lors, cette phrase peut être prononcée en n'importe quel lieu, car son sens est détaché de la deixis liée à la situation de parole.

Par cet exemple, on peut constater, pour ajouter une précision aux remarques précédentes, que Bühler avait de fait découvert une partie de la déicticité de la forme verbale. Mais son analyse reste trop limitée au spatial et n'arrive pas à s'ouvrir au temps.

\subsection{L'organisation des "systèmes déictiques »}

Lorsque l'on parle à l'heure actuelle de deixis ego hic nunc, on fait, sans le savoir, implicitement référence à Bühler, qui est à l'origine de cette distinction entre plusieurs classes de déictiques - que l'on retrouve de manière assez générale à travers les langues.

En effet, Bühler a distingué trois grandes classes de déictiques :

- les déictiques de la personne : en français je et tu, auxquels il faut associer nous et vous, qui ne présentent absolument pas les caractéristiques du pluriel nominal ;

- les déictiques de l'espace : en français ici, là et là-bas;

- les déictiques du temps: en français maintenant et quelques autres prenant appui sur jour: aujourd'hui, hier, demain.

À ces trois classes fondamentales, Bühler adjoint également les déictiques de manière (ainsi) et les démonstratifs (ce, cet, celui-ci, celui-là).

D'autre part, Bühler a également mis en évidence - ce qui est très important pour l'épistémologie linguistique - que tout système déictique présente cette particularité d'avoir un centre, un point zéro, une origo, à partir de laquelle se développe un microsystème à chaque fois spécifique. Ces "systèmes déictiques", qui sont la grande conquête de Bühler, sont centrés sur une origo, qui coïncide naturellement avec la personne et les coordonnées du locuteur. 


\subsection{Le fonctionnement de la deixis selon Bühler}

38

le vaste "champ monstratif " ainsi (déc)ouvert par lui, Bühler distingue trois types généraux de monstration selon leur mode d'exercice et leur champ d'application :

1. la « demonstratio ad oculos » (en latin dans le texte), qui correspond à une deixis directe, c'est-à-dire ostensive, dans la situation de discours et qu'il serait possible de relier aux travaux de Wittgenstein sur l'ostension et la démonstration dans les Philosophische Untersuchungen (1953, mais bien antérieures dans leur conception). Le champ monstratif est constitué par l'espace commun ouvert à la perception, au sens large, du locuteur et de l'auditeur.

2. l'anaphore et la cataphore, terme précisément créé par Bühler (1934: 121s, note 1), opèrent par transposition sur un champ monstratif représenté par le texte et son contexte.

3. la "deixis imaginative ", expression là encore créée par Bühler (Deixis am Phantasma) pour rendre compte d'un fonctionnement particulier de la deixis : celui où elle s'applique au «domaine de l'évocable absent» et au "domaine de l'imagination constructive » (1934: 124s) et où elle s'exerce donc essentiellement sur des représentations intérieures.

Il faut insister sur quelques observations personnelles, qui soulignent des aspects fondamentaux concernant le fonctionnement des trois types de deixis :

1. ce sont les mêmes outils linguistiques qui fonctionnent dans les trois domaines distingués par Bühler, ce qui confirme la grande unité du champ monstratif. Ainsi en allemand, es peut exercer aussi bien les fonctions de cataphorique que d'anaphorique et dieser aussi bien celles de déictique que d'anaphorique.

2. la deixis n'est pas seulement geste ostensif, elle s'intègre à la valeur syntactico-sémantique de l'énoncé et accède à des fonctions beaucoup plus essentielles, comme nous l'avons indiqué trop rapidement (1992:370s).

Reprenant un exemple cité en allemand par K. Brugmann (1904): Donne-moi ce chapeau!, K. Bühler distingue dans ce chapeau trois éléments constitutifs de la relation déictique : le geste du doigt, le déictique ce et le lexème chapeau. Évoquant ensuite trois possibilités de regroupement binaire ; ou " geste + déictique ", ou " geste + chapeau », ou "déictique + chapeau», il constate simplement qu'il est difficile de se passer d'un support lexical sur lequel précisément "geste + déictique » effectuent la visée ostensive. Or, il est possible de pousser plus loin l'analyse de Bühler en soulignant dans cet exemple l'association d'un argument (chapeau) et d'un prédicat déictique (ce), ce qui peut également se réaliser sous la forme suivante: le chapeau que voilà. Nous venons ainsi de démontrer que le déictique ce se comporte comme un véritable prédicat - ce qui ne saurait surprendre quand on connaît les prédicats déictiques en gotique :

got. hiri « toi, (viens) ici ! ; got. hirjats « vous deux, (venez) ici !»

41 Ces formes (verbales) sont naturellement issues du déictique got. hêr « ici ».

1. Bühler a raison d'insister sur l'aspect mémoriel (Bühler emploie le terme de topomnestisch 1934, 147s) que comporte tout phénomène de reprise. En effet, le principe de l'anaphore ne met pas seulement en jeu un processus linguistique de pure reprise du contenu d'un groupe, mais elle comporte dans son fonctionnement une dimension mémorielle qui dépasse nettement le cadre du langage, comme l'ont montré Yule et Brown (1983) avec l'exemple célèbre du poulet. 


\subsection{Cas particuliers de deixis et d'anaphore}

42 À la suite d'un raisonnement très simple, Bühler en vient à se poser la question suivante: puisque les indices remplacent les noms, pourquoi l'inverse ne serait-il pas attesté et pourquoi n'y aurait-il pas dans les langues des noms employés à la place des indices déictiques? Pour démontrer la justesse de son raisonnement, Bühler se met en quête d'exemples auprès de collègues qui lui signalent des cas de ce genre dans les langues amérindiennes et en japonais.

Or, il n'est pas nécessaire d'aller chercher si loin, car des exemples existent dans certaines langues indo-européennes (cf. Rousseau 1993: 324s). En gotique, langue germanique $\mathrm{du} \mathrm{IV}^{\mathrm{e}}$ siècle, les formes fléchies (avec des morphèmes spécifiques de cas locaux) de dals « vallée » déterminent toujours la position basse par rapport au locuteur ou au narrateur :

L 4, 9: wairp buk papro dalap « jette-toi d'ici en bas!»

Dans cet exemple, comme dans d'autres, le lexème dal- est dépourvu de toute valeur référentielle, car il n'y est jamais question d'une "vallée »; en outre dalap fonctionne dans les mêmes conditions que le déictique papro " d'ici, de là ». Ce sont précisément ces conditions de fonctionnement que Bühler assignait a priori à ce qu'il a appelé les "prodémonstratifs ». Ce fonctionnement s'est conservé jusque dans l'expression zu Tal " abwärts », dont H. Paul signale la désuétude vers 1900.

Parallèlement à ce phénomène et de manière complémentaire, Bühler a noté que « tout signe monstratif était susceptible d'exercer une fonction de dénomination; sinon il n'existerait pas de pronoms" (1934: 143). Il souligne également qu'il n'y a pas de coupure nette entre "mots monstratifs» et "mots dénominatifs", car l'anaphore établit un pont entre ces deux classes. Elle est donc, selon Bühler, «du point de vue sématologique la coopération ou le rapprochement fonctionnel le plus remarquable concernant ces deux classes de mots » $(1934: 300)$.

\section{La subjectivité dans le langage}

À partir du «champ monstratif ", qui lui fournit la base de départ théorique solide et incontestable et qui représente à première vue l'aspect le plus innovant de la Sprachtheorie, Bühler va poser au fil des pages de son ouvrage les linéaments essentiels d'une théorie beaucoup plus vaste de la subjectivité, sans toutefois vraiment la développer. Cette théorie est fondée non pas tant sur l'existence de classes nouvelles spécifiques dans la langue - comme par exemple la reconnaissance des modalisateurs, des appréciatifs ou des particules illocutoires, qui avec les déictiques représentera, bien après Bühler, l'ossature des phénomènes relevant de la subjectivité - mais essentiellement sur les fonctions du langage, dont Bühler reste un des spécialistes incontestés et qui se traduisent par divers faits de langue, comme le choix des mots et la construction de la phrase (1934: 73).

On reproche précisément parfois à Bühler d'avoir omis au moins deux fonctions du langage, que l'on retrouve chez Jakobson (1960) : la fonction dite " phatique », qui vient tout droit de B. Malinowski (1923) et de ce qu'il appelait la " communion phatique », et la fonction dite "poétique ", qui est un apport personnel de Jakobson. Nous pensons 
que l'intersubjectivité bühlérienne est un concept qui mène à la fonction phatique et que Bühler n'a surtout pas ignoré la spécificité de la poésie dans son ouvrage.

\subsection{Le behaviorisme et son dépassement chez Bühler}

Il nous semble incontestable qu'il existe chez Bühler des aspects de sa pensée qui relèvent d'un type d'analyse de nature behavioriste. À commencer par le fonctionnement du modèle de l'organon :

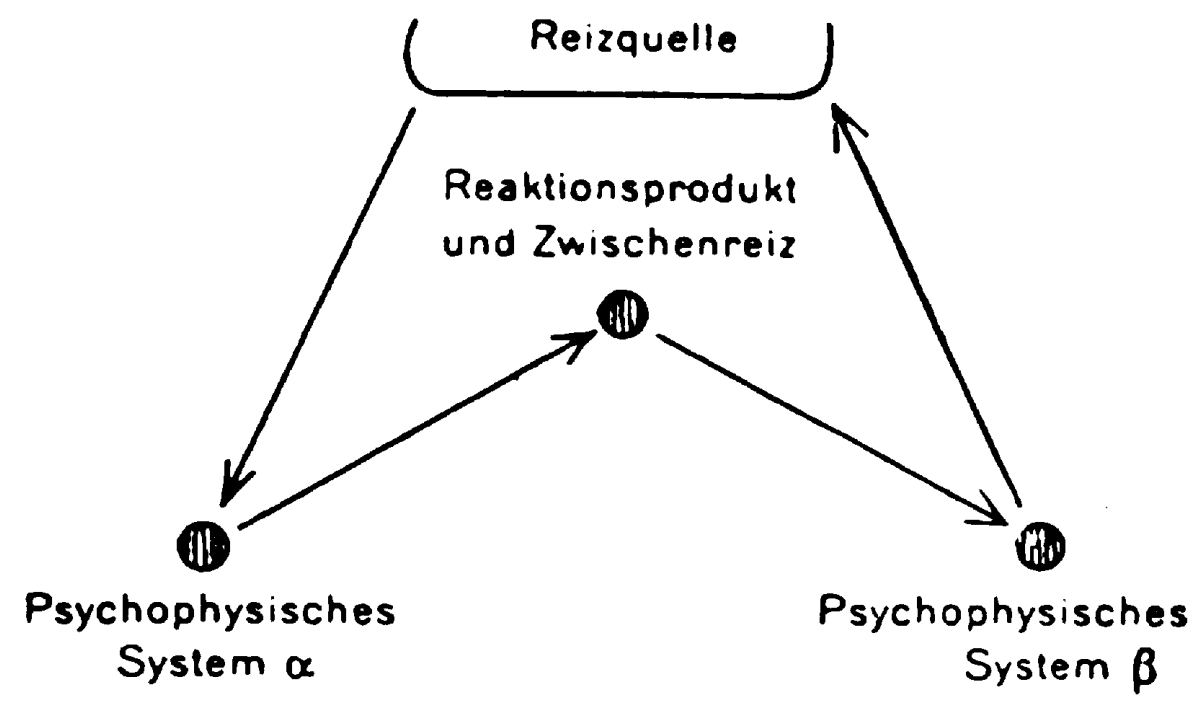

(1934:26)

Karl Bühler a recours à l'expérience banale suivante, illustrée par le schéma ci-dessus, où deux personnages $\mathrm{A}$ et $\mathrm{B}$ se trouvent dans une pièce :

- À entend le bruit de la pluie contre les vitres et dit à B : « Il pleut »;

- entendant ces mots, $\mathrm{B}$ se lève et regarde à travers la fenêtre.

Ainsi Bühler considère qu'il y a eu un enchaînement d'événements, parmi lesquels il identifie le bruit de la pluie comme :

- la source de stimulation (Reizquelle) pour A

et les mots « Il pleut » dans leur double rôle, à la fois :

- produit de la réaction (Reaktionsprodukt) pour A

- nouvelle source de stimulation (Zwischenreiz) pour B.

Même s'il n'est pas habituel de classer Bühler parmi les behavioristes, on se doit de faire observer qu'il utilise ici le schéma type du behaviorisme classique: stimulusréponse.

Il faut ajouter que certains commentateurs de Bühler l'ont tiré encore davantage vers le behaviorisme, comme par exemple H. Hörmann (1971) lorsqu'il cherche à expliciter les trois fonctions de Bühler par la mise en relation systématique d'un comportement avec la perception de certains sons - ce que Bühler n'a jamais formulé sous cette forme. 
Pour nous en tenir au schéma précédent et à son fonctionnement de type behavioriste, une comparaison avec un passage de Language (1933) de L. Bloomfield est tout à fait révélatrice. Bloomfield se place dans la situation suivante : « Supposons que Jack et Jill descendent un sentier. Jill a faim. Elle voit une pomme sur un arbre. Elle fait un bruit avec son larynx, sa langue et ses dents. Jack saute la barrière, grimpe à l'arbre, prend la pomme, l'apporte à Jill, la pose dans sa main. Jill mange la pomme.» (p. 26-29 de la trad. fr.)

Dans le commentaire qu'il donne de cette scène, Bloomfield décompose la succession des événements décrits ci-dessus en trois actions principales :

A - les actions pratiques précédant l'acte de parler

B - le discours

C - les actions pratiques suivant l'acte de parler.

Nous retrouvons exactement la même organisation de la situation que celle décrite par Bühler à propos de la pluie :

A - un fait du monde extérieur est source de stimulation

B - le discours est considéré à la fois comme réaction au stimulus précédent et comme stimulus intermédiaire

$\mathrm{C}$ - ce stimulus a pour conséquence à son tour une réaction, qui se traduit par un comportement.

À s'en tenir à cette comparaison, il est clair que Bühler et Bloomfield abordent les faits linguistiques exactement de la même manière, par le biais d'une expérience comportementale.

Mais il y a plus: le sens est mis délibérément entre parenthèses au profit du comportement. De manière identique chez l'un et chez l'autre, la distinction est faite entre deux types de comportement :

- un comportement physique (les actions)

- un comportement linguistique (le discours),

le premier permettant d'appréhender le second et surtout prenant peu à peu et insidieusement sa place (cf. le discours considéré comme un bruit chez Bloomfield). En effet, un behavioriste rigoureux comme J.-B. Watson (1924) est catégorique à ce sujet : « Du point de vue behavioriste, le problème du sens est une pure abstraction. Il ne se pose jamais dans l'observation scientifique du comportement. Nous observons ce que l'animal ou l'homme fait. Ce qu'il "veut dire", c'est ce qu'il fait. » (1924: 354-355). En d'autres mots, le sens est réduit ou ramené à du comportement. En effet, par un conditionnement de type pavlovien, le signe linguistique n'est pas autre chose qu'un stimulus, qui provoque le même comportement que celui suscité à l'origine par l'objet ou la chose elle-même. On retrouve ici le principe scolastique qui sous-tend l'organon : " verbum stat pro re ". Et même des idées chères à Peirce et au pragmatisme moderne, selon lesquelles le sens ne serait rien d'autre qu'une sorte de consigne transmise à l'utilisateur pour agir de telle ou telle manière.

73 Mais Bühler, même s'il peut donner l'impression d'adopter dans l'organon une attitude behavioriste, se sépare nettement du behaviorisme ambiant à son époque sur au moins deux points essentiels : d'abord il cherche à fonder une véritable théorie du sens, à propos de laquelle il prend ses distances par rapport à Husserl, qui avait proposé de la 
fonder uniquement à partir des actes (1934:69); ensuite, cette théorie du sens essaie de maintenir un équilibre entre facteurs sociaux et facteurs subjectifs - comme cela apparaît déjà dans la Krise der Psychologie (1927) : certes, « il est impossible de constituer le concept de sens sans faire appel à des intentions et à une référence au sujet ». Mais Bühler ajoute immédiatement : «Les choses et les événements du monde sont pour le sujet qui les vit trop chargés de sens pour qu'il soit capable - correctement ou faussement - d'en retirer quelque chose ou de leur imputer quelque chose, mais même seulement de les situer» $(1927: 132)$. Et il rappelle à nouveau cette position avec force dans la Sprachtheorie: "...on est obligé de considérer que la théorie de la structure traditionnellement dérivée du modèle authentique de l'Organon dans le langage et par conséquent du traitement objectif de la langue et également le facteur social de la langue ont logiquement la priorité ou au moins la parité vis-à-vis d'une théorie des actes rapportée au sujet » (1934:69).

La théorie de la signification développée par Bühler cherche en particulier à répondre à un point précis, toujours considéré comme délicat: comment s'associent les unités significatives dans les langues? Nous avons toujours considéré pour notre part et indépendamment de Bühler qu'il ne saurait s'agir d'une addition mathématique des éléments, mais qu'il existe une hiérarchie dans les regroupements, qui s'exerce de manière progressive (schrittweise).

Posant le problème du passage et de la relation des mots à l'« unité phrastique » (selon son expression $1934: 257$ ), il propose une hypothèse explicative tout à fait intéressante. En effet, considérant que les signes sont non pas des invitations à l'action ou des marques de réaction, comme les conçoit le behaviorisme, mais des éléments chargés de sens, faisant l'objet d'une véritable "mise en sens » et d'une compréhension, c'est-àdire d'un encodage et d'un décodage sémantiques, Bühler fait appel à la notion de «sur-sommativité » ou "sommativité majorante » (all. Übersummativität), conçue par Christian von Ehrenfels (1859-1932) en 1890 dans un article, intitulé Über Gestaltqualitäten et publié dans la Vierteljahresschrift für wissenschaftliche Philosophie, dont le principe est formulé en ces termes: «Le tout représente plus que l'addition de ses parties ». Ce premier principe est lui-même pondéré par son homologue, celui de la "sous-sommativité » ou "sommativité minorante» (all. Untersummativität). Ces deux principes sont appliqués par Bühler à l'étude des mots composés et à celle de la métaphore. Cette méthode d'analyse, qui est aussi appelée le «modèle du double filtre » $(1934: 355)$ revient finalement à pratiquer une sélection sémantique alternative et réciproque. Nous sommes effectivement très loin du behaviorisme.

\subsection{Théorie du sujet et intersubjectivité}

La théorie du sujet, qu'on était en droit d'attendre de Bühler, n'est au fond qu'à peine esquissée dans la Sprachtheorie. Pour la formuler explicitement, il faut faire appel à plusieurs passages de l'ouvrage; et cela ne suffit pas.

Bühler disposait en effet de tous les éléments constitutifs indispensables à cette théorie, et notamment des deux piliers fondamentaux :

- dans la construction « carrée » établie par Bühler (1934: 49), le langage apparaît divisé en deux : d'un côté, tout ce qui relève de la langue (Sprachwerk et Sprachgebilde); de l'autre, tout ce qui est affaire du sujet (Sprechhandlung et Sprechakt). Incontestablement Bühler avait senti 
ce va-et-vient, ce rapport dialectique entre sujet et langue, comme l'attestent de nombreuses pages du livre. Benveniste dans un article du Journal de Psychologie: De la subjectivité dans le langage (1958). Comme à son habitude, Benveniste ne cite aucune référence, ne renvoie à aucun auteur. Or, il est sûr que Benveniste connaissait la Sprachtheorie de Bühler; en effet, dans un article de 1954, paru dans le Journal de Psychologie et ayant pour titre Tendances récentes en linguistique générale, Benveniste cite expressément le « recueil publié en 1933 par le Journal de Psychologie sous le titre Psychologie du Langage", dans lequel se manifeste " un renouveau éclatant des vues théoriques et des affirmations doctrinales " (1954). Ce recueil d'articles importants contient précisément un chapitre de la Sprachtheorie de Bühler (aux pages 101-119), traduit en français par Geneviève Bianquis. Comment admettre que l'ouvrage si novateur de Bühler n'ait pas attiré l'attention de Benveniste et n'appartienne pas à ce « renouveau éclatant des vues théoriques » dont il parle?

Relu sous cet éclairage, l'article de Benveniste exhibe en effet plus d'une parenté avec les thèses fondamentales de Bühler; reprenons quelques passages de l'article de Benveniste qui dénotent une imprégnation certaine de Bühler :

1. « on ajouterait que le comportement du langage admet une description behavioriste, en terme de stimulus et de réponse, d'où l'on conclut au caractère médiat et instrumental du langage » (p. 258)

2. «c'est dans et par le langage que l'homme se constitue comme sujet; parce que seul le langage fonde [...] dans sa réalité [...] le concept d'“ego" ». (p. 259)

3. « la conscience de soi n'est possible que si elle s'éprouve par contraste. Je n'emploie je qu'en m'adressant à quelqu'un, qui sera dans mon allocution un tu ». (p. 260)

4. « on est en présence d'une classe de mots, les "pronoms personnels", qui échappent au statut de tous les autres signes du langage ». (p. 261)

Les lecteurs de Bühler et de Benveniste pourront certainement faire bien d'autres rapprochements. Mais, heureusement, l'article de Benveniste ne se limite pas à ces passages issus de Bühler. Benveniste a le mérite, certes après Russell (1940), d'intégrer d'une part le temps verbal et la temporalité humaine et d'autre part les « énonciations performatives » (type je jure) dans le domaine de la subjectivité propre au langage.

Mais, pour en rester à cette notion de "subjectivité", les conclusions un peu sommaires de Benveniste laissent le lecteur sur sa faim quant aux conditions mêmes dont celle-ci s'exerce dans le langage: "Le langage est donc la possibilité de la subjectivité, du fait qu'il contient toutes les formes appropriées à son expression; et le discours provoque l'émergence de la subjectivité du fait qu'il consiste en instances discrètes. Le langage propose en quelque sorte des formes "vides" que chaque locuteur 
en exercice de discours s'approprie et qu'il rapporte à sa "personne", définissant en même temps lui-même comme je et un partenaire comme tu » (p. 263).

Et in fine, Benveniste élargit la subjectivité à l'« intersubjectivité » - ce qui nous ramène une fois de plus tout droit à Bühler. En effet, nous pensons que si Bühler n'a qu'à peine esquissé ce qui aurait pu être une grande théorie de la subjectivité, vers laquelle le conduisaient ses prémisses, c'est que celle-ci a été reprise et même absorbée par un principe dominant de la Sprachtheorie: l'intersubjectivité, qui n'est au fond qu'une subjectivité à coloration sociale. Cette vue est confirmée par plusieurs passages de la Sprachtheorie, où il s'appuie sur le phénomène capital de l'intersubjectivité.

Certes, il donne l'impression d'opposer nettement dans son modèle "carré », bâti sur une double dichotomie, les "phénomènes subjectifs" aux "phénomènes nonsubjectifs, c'est-à-dire fixés de manière intersubjective " (1934: 49). Mais quelques pages plus loin, il reprend à son compte les grands principes saussuriens et notamment «le caractère intersubjectif des structures de la langue et, par conséquent, leur indépendance par rapport au locuteur appartenant à une communauté linguistique " (1934: 58), rappelant à ce propos les paroles de Saussure : « la langue est extérieure à l'individu, qui à lui seul ne peut ni la créer, ni la modifier; elle n'existe qu'en vertu d'une sorte de contrat passé entre les membres de la communauté » (CLG : 31). À plusieurs reprises, Bühler s'appuie dans ses démonstrations sur l'intersubjectivité inhérente au langage humain. À l'image de la navette proposée par Platon dans le Cratyle, il fait correspondre les « conventions de la langue régulées de manière intersubjective" (1934: 68). Enfin, une expression comme celle du «circuit intersubjectif des signes » $(1934: 231,301)$ est placée axiomatiquement au sommet de son édifice théorique.

\subsection{La subjectivité dans la littérature}

Les passages que Bühler consacre aux arts en général (par ex. la peinture) et à la littérature en particulier s'inscrivent dans le cadre plus vaste d'une sématologie (terme qu'il préfère à séméologie 1934 : 9), à laquelle pourtant il ne consacre aucun chapitre particulier, mais dont les éléments sont épars au fil des pages. Le premier travail consiste à retrouver quelques principes essentiels de cette théorie.

L'axiome sur la nature sémiologique de la langue ouvre la voie à la sématologie, mais pas à n'importe laquelle. En effet, "nous constatons que toute tentative pour construire une sématologie sur des bases purement matérielles est une tentative avec des moyens inadéquats, qui dès les faits les plus simples de l'échange des signes linguistiques est condamné à échouer ou du moins à se bloquer» (1934: 225). Cela s'explique, sans que Bühler l'exprime directement en ces termes, par le fait que la sématologie dépend fondamentalement du sujet et que son fonctionnement est l'objet de choix subjectifs ou intersubjectifs. Bühler le démontre par le fonctionnement du principe de pertinence :

88 «C'est un principe général de la sématologie que toutes les choses ou tous les processus dans le monde que nous utilisons comme signes, sont utilisés selon le principe de la pertinence sélective (abstraktive Relevanz). Quand on introduit par exemple des signaux lumineux dans la circulation des bateaux, des trains ou des voitures, les conventions ont les valeurs suivantes : rouge $\rightarrow$ danger, voie fermée; vert $\rightarrow$ pas de danger, voie 
libre » (1934: 224). Ces valeurs ne sont reconnues comme telles que parce qu'elles sont le résultat de conventions définies par un large accord.

Que devient dans ce contexte la littérature et, plus particulièrement, la poésie lyrique ? À la page 55 de sa Sprachtheorie, Bühler cite quelques vers célèbres du poème Gefunden de Goethe :

Ich ging im Walde

So für mich hin,

Und nichts zu suchen,

Das war mein Sinn.

Et s'interrogeant sur le thème de cette poésie, il affirme : "Une théorie linguistique large doit également faire place dans son système à cette branche de la recherche linguistique » (1934:55). Or, nous pensons que la théorie de Bühler, telle qu'elle figure dans la Sprachtheorie, n'est pas en mesure de rendre compte, malgré la bonne volonté évidente de son auteur, de textes poétiques. En étant parfaitement conscient du risque de l'entreprise hardie, nous voudrions tenter de montrer de quelle manière et dans quel sens il faut adapter et surtout renforcer les théories de Bühler pour les rendre aptes à proposer une solution pour l'interprétation des textes poétiques.

91 Modifiant la classification des signes de Peirce (1932), établie sur d'autres critères et distinguant icône, indice et symbole, Bühler développe une analyse nouvelle, reconnaissant trois valeurs particulières du signe :

1. dans la mesure où le phénomène Ph renvoie aux «objets " et aux «états de choses » (Darstellungsfunktion), le signe est un symbole;

2. dans la mesure où il exprime l'état intérieur du locuteur (Ausdrucksfunktion), le signe est un symptôme;

3. dans la mesure où il représente un appel pour l'auditeur, guidant son comportement (Appellfunktion), le signe est un signal.

Le «signe-symptôme" ne se rencontre-t-il que dans la poésie lyrique? Bühler reconnaît tout à fait la spécificité de la poésie : «Le lyrisme et la rhétorique ont chacun quelque chose de particulier en eux-mêmes ». Mais il ajoute aussitôt: "il n'est pas nécessaire d'aller seulement vers le poète lyrique pour découvrir la "fonction expressive » en tant que telle; certes, la moisson sera beaucoup plus riche chez celui-ci. Et s'il s'agit d'un poète lyrique à l'écriture tout à fait originale, il écrit parfois au-dessus de la porte ; le logicien doit rester dehors " $(1934: 32)$. Cette évocation du logicien à propos de la poésie n'est pas unique chez Bühler. Un autre passage lui fait écho, faisant allusion au mathématicien et au "logisticien ", quand Bühler cite cette phrase, qui doit être une poésie connue ou un refrain à la mode : «Zwei Augen, ach zwei Augen, die kommen mir nicht aus dem Sinn », qu'il commente en ces termes : « Les logisticiens ont l'habitude de renvoyer de telles phrases hors du domaine de la science vers celui du "lyrisme»; et pourtant il n'est pas permis de dénier à de telles énonciations toute valeur de représentation objective du seul fait que le signe monstratif ich (dans mir) y apparaît » (1934 : 372).

Ce commentaire rappelle bien évidemment l'attitude du mathématicien et logicien Gottlob Frege (1848 - 1925), dont Bühler ne cite pourtant jamais le nom dans sa Sprachtheorie (ce qui prouve à quel point Frege restait encore un inconnu en 1934 !) et qui était partisan d'une solution beaucoup plus radicale, conforme à sa propre théorie du sens et de la référence, qu'il résume ainsi dans les Ausführungen über Sinn und Bedeutung (écrites entre 1892 et 1895) : «Dans la poésie, les mots ont seulement un sens, 
mais dans la science et partout où la question de la vérité nous préoccupe, nous ne nous contenterons pas du sens, mais nous associerons aussi une référence (Bedeutung) aux noms propres et aux mots exprimant un concept». Ces propos sont clairs : Frege soutient la thèse que ce serait l'effet de la subjectivité de priver les noms de référence en poésie. Une telle position est-elle admissible? Est-elle même conciliable avec la théorie de Bühler?

Certes, et il faut le reconnaître, Bühler ne semble jamais se situer dans le cadre « sensréférence » cher à Frege, tout simplement peut-être parce qu'il ignorait les travaux de Frege. Si Bühler traite souvent du sens ou de la signification (Bedeutung), il n'évoque pratiquement jamais la notion de référence (all. Bezug ou Referenz), sauf peut-être dans une expression soulignée, " die gegenständliche Bedeutung ", qu'il glose précisément par "Zuordnung " et que l'on pourrait rendre en français par "référence matérielle " (1934: 34). Le fait de n'avoir pas développé cette notion juste entrevue est certainement une lacune fondamentale pour la théorie de Bühler (voir Rousseau, à paraître b, § 1.2.), celle qui l'empêche d'avoir la théorie globale des faits de langage dont il rêvait, y compris des phénomènes poétiques.

Or il nous semble possible de marier avec une hardiesse épistémologique mesurée nous en convenons volontiers - la théorie de Frege sur sens et référence avec la théorie de Bühler sur les trois fonctions du signe linguistique, qui apparaissent dès lors comme un enrichissement considérable de la Bedeutung frégéenne. Cette conception unifiée et hardie de deux penseurs apparemment si différents peut se traduire par le schéma suivant :

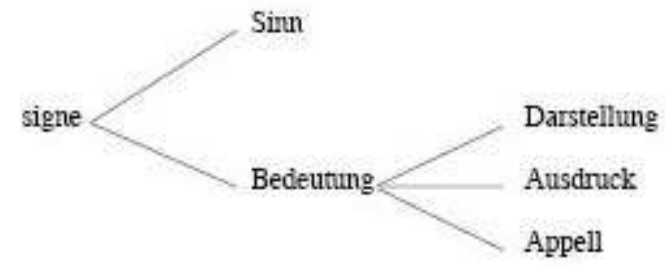

En effet, les trois valeurs distinguées par Bühler, le symbole, le symptôme et le signal, sont au fond des valeurs référentielles. Il existe donc au fond deux grands types de référence :

- une référence « objective », illustrée par la Darstellungsfunktion;

- une référence intersubjective, qui garantit la première et qui est représentée par l'axe Je-Tu de Martin Buber.

Dès lors, la poésie a sa place dans ce schéma ; elle possède à la fois un sens, mais aussi une référence (ce que reconnaissait Bühler mais que niait Frege), qui est entièrement subjective.

Roman Jakobson est le linguiste qui a poussé plus loin l'analyse de Bühler sur les fonctions du langage, mettant notamment en évidence la fonction poétique. Or, il ne semble pas qu'il y ait la moindre contradiction entre l'interprétation que nous proposons ici et les deux thèses essentielles que défend R. Jakobson :

-Jakobson soutient, dans la ligne de Frege, que le sens, même en poésie, est objectivement identifiable dans et par la langue ;

- Jakobson déclare en 1960 qu'en poésie « la suprématie de la fonction poétique sur la fonction référentielle n'oblitère pas la référence, mais la rend ambiguë». 

loin la problématique.

\section{BIBLIOGRAPHIE}

BENVENISTE, Émile (1958) : «De la subjectivité dans le langage », in : Journal de Psychologie 55, p. 258-266.

BLommFIELD, Leonard (1933) : Language, New York, Holt, Rinchart \& Winston.

BüHLER, Karl (1920) : « Kritische Musterung der neueren Theorien des Satzes », in :

Indogermanisches Jahrbuch 6, p. 1-20.

BÜHLER, Karl (1927) : Die Krise der Psychologie, Jena, Fischer.

BÜHLER, Karl (1933) : « Die Axiomatik der Sprachwissenschaften », in : Kantstudien 38, p. 19-90.

BÜHLER, Karl (1933) : «L'onomatopée et la fonction représentative du langage », in : Psychologie du Langage, Paris, Alcan.

BÜHLER, Karl (1934) : Sprachtheorie, Jena, Fischer.

EHRENFELS, C. (1890) : Über Gestaltqualitäten in Vierteljahresschrift für wissenschaftliche Philosophie 14, p. 249-292 (notamment p. 260).

Germanica, 26 | 2000 
EsCHBACH, Achim Hrsg., (1984) : Bühler-Studien, 2 Bde. Frankfurt a. M., Suhrkamp.

EsCHBACH, Achim Ed. (1988) : Karl Bühler's Theory of Language, Amsterdam/Philadelphia, John Benjamin.

FREGE, Gottlob (1892-1895) : « Ausführungen über Sinn und Bedeutung », in : Nachgelassene Schriften (Hrsg. von G. Gabriel, H. Hermes, F. Kambartel, F. Kaulbach) Hamburg, F. Meiner Verlag, 1969.

GRAUMANN C.F./HERRMANN Th. Hg. (1984) : Karl Bühlers Axiomatik, Frankfurt a.M., V. Klostermann.

HöRMANN, Hans (1971): Psychologie der Sprache, Berlin/Heidelberg, Springer.

JAKOBSON, Roman (1960) : « Linguistics and poetics », in : Sebeok T.A. ed. Style in Language, New York, John Wiley, p. 350-377.

RousSEAU, André (1992) : «La deixis : un problème de logique et de philosophie du langage », in : La Deixis (M.A. Morel/L. Danon-Boileau éd.). Paris, P.U.F., pp. 365-374.

ROUSSEAU, André (à paraître a) : « La théorie syntaxique de Karl Bühler », in : L'Histoire de la syntaxe de 1870 à 1940, (A. Rousseau éd.) 20 pages.

RoUSSEAU, André (à paraître b) : « Réflexions théoriques et historiques sur la théorie de Bühler : les "systèmes déictiques" et les cas locaux ", in : Mélanges pour Jean Perrot, 10 pages.

RusSEL, Bertrand (1940) : An Inquiry into Meaning and Truth, London, Mac Millan (trad. française: Signification et vérité, Paris, Flammarion, 1958).

TRoubETZKoY, Nicolas (1939) : « Grundzüge der Phonologie », in : TCLP 7.

WUNDERLICH, Dieter (1969) : «Karl Bühlers Grundprinzipien der Sprachtheorie », in : Muttersprache 79, p. 52-62.

\section{RÉSUMÉS}

Karl Bühler (1879-1963) reste encore actuellement peu connu malgré ses nombreuses publications à l'interface de la psychologie et de la linguistique. Sa Sprachtheorie (1934) représente son ouvrage majeur, qui s'ouvre par une Axiomatique, où sont exposées quatre thèses essentielles (dont celle de l'Organon et des trois fonctions : représentation, expression, appel) et qui développe ensuite une division en deux champs de l'étude du langage : " champ monstratif » et «champ symbolique ». Il a fondé une véritable théorie moderne de la deixis, qui cherche à identifier les systèmes déictiques à partir d'une origo (ego, hic, nunc), à en distinguer les types de fonctionnement (demonstratio ad oculos, Deixis am Phantasma, anaphore et/ou cataphore, terme qu'il crée), et surtout à montrer les passerelles possibles entre les deux champs (notamment par le phénomène important des «prodémonstratifs»). Bühler disposait de tous les éléments pour construire la théorie attendue de la subjectivité dans le langage; mais son orientation vers la psychologie sociale l'a conduit davantage vers l'intersubjectivité et c'est un Benveniste, imprégné de Bühler, qui développera cette théorie en 1958. Toutefois Bühler revient à plusieurs reprises sur la subjectivité, notamment à propos des arts et de la littérature, ce qui nous conduit à rectifier sa théorie d'une manière inattendue : en y introduisant la référence de Frege - qui permet in fine de situer les trois fonctions de Bühler dans un cadre théorique solide et homogène.

Trotz seiner zahlreichen Veröffentlichungen auf dem Gebiet zwischen Psychologie und Linguistik ist Karl Bühler (1879-1963) derzeit immer noch wenig bekannt. Sein bedeutendstes Werk, seine 
Sprachtheorie (1934), beginnt mit einer Axiomatik, in der vier Thesen vertreten werden - unter anderem die des Organonmodells und der drei Funktionen : Darstellung, Ausdruck, Appell. Da werden auch zwei Felder der Sprachbetrachtung entwickelt : Zeigfeld und Symbolfeld. Er hat eine wirklich moderne Theorie der Deixis gegründet, die versucht, die deiktischen Systeme von einer Origo (ego, hic, nunc) ausgehend $\mathrm{zu}$ identifizieren und deren Funktionsweisen $\mathrm{zu}$ unterscheiden (demonstratio ad oculos, Deixis am Phantasma, Anapher und/oder Katapher - ein von ihm geprägter Terminus) und vor allem die denkbaren Übergänge zwischen beiden Feldern zu zeigen (insbesondere anhand des wichtigen Phänomens der Prodemonstrativa). Bühler verfügte über alle Elemente, um die erwartete Theorie der Subjektivität in der Sprache auszubauen; aber seine Ausrichtung auf eine Sozialpsychologie hat ihn eher in Richtung Intersubjektivität gelenkt. Erst 1958 wird ein von Bühler stark beeinflußter Benveniste diese Theorie entwickeln. Insbesondere im Bereich der Kunst und der Literatur hat doch Bühler mehrmals die Subjektivität wieder aufgegriffen - und gerade das führt uns überraschend dazu, seine Theorie richtigzustellen: Indem wir die Fregesche Referenztheorie einführen, die letztendlich erlaubt, die drei Funktionen Bühlers in einem theoretisch soliden und homogenen Rahmen unterzubringen.

\section{AUTEUR}

\section{ANDRÉ ROUSSEAU}

Université Charles de Gaulle - Lille III 